\title{
The immediate effects of foam roller with vibration on hamstring flexibility and jump performance in healthy adults
}

\author{
Jae-Heon Lim', Chi-Bok Park²,* \\ ${ }^{1}$ The Lab Cooperative, Gwangju, Korea \\ 2Department of Physical Therapy, Nambu University, Gwangju, Korea
}

This study aimed to determine whether foam roller with vibration (FRV) can improve hamstring flexibility and jump performance in young adults. Twenty subjects were randomly allocated into either the FRV or the foamroller with no vibration (FRNV) groups. The vibration of the vibration foam-roller was $32 \mathrm{~Hz}$. All participants completed one 10-min session on the hamstring. Active straight leg raising (ASLR) test in supine position and active knee extension (AKE) test in sitting position were performed to measure hamstring flexibility, before and immediately after the session. ASLR, AKE, and vertical jump performance test were per-

\section{INTRODUCTION}

A warm-up exercise improves blood circulation, exercise performance, and prevents injury. Warm-up is a kind of exercise to induce the body toward a suitable state before exercising. There are many warm-up exercises, including fascia release, stretching, walking, running, biking, gymnastics, etc. (Bishop, 2003).

Fascia is a connective tissue surrounding the muscles, nerves, and blood vessels, and it connects the structures of the body. It can be limited due to injury, disease, inactivity or inflammation. These limitations can reduce flexibility, strength, endurance, and coordination (Schroeder and Best, 2015). Moreover, it can also cause a great deal of physical pain. The studies about the effects of fascia relaxation and stretching techniques on the range of motion and response time reported that fascia relaxation increased flexibility of quadriceps and hamstrings (Kuruma et al., 2013; Škarabot et al., 2015). formed before the session and immediately after the session. Analysis of covariance was used to determine statistical significance. ASLR and AKE tests were significantly different between FRV and FRNV, except the right hamstring. There was significant improvement with respect to ASLR and AKE tests after the session in FRV group. The vertical jump performance test of both groups was not significantly different after the session. FRV is more effective than FRNV for improving hamstring flexibility.

Keywords: Foam-roller, Vibration, Hamstring, Flexibility
${ }^{*}$ Corresponding author: Chi-Bok Park (D) https://orcid.org/0000-0003-3791-7966 Department of Physical Therapy, Nambu University, 23 Cheomdanjungang-ro, Gwangsan-gu, Gwangju 62271, Korea

E-mail: oasislovept@hanmail.net

Received: November 11, 2018 / Accepted: January 21, 2019
Recently, in the field of exercise rehabilitation and fitness conditioning, the use of foam roller for fascia relaxation has been on the rise for the purpose of exercise preparation and recovery of muscle functions (Peacock et al., 2015). Foam rollers are used in diverse fields, including sports rehabilitation and exercise. Foam rollers utilizes the rolling properties to lean against the body and then move the body. It is used to relax the fascia and eliminate tension of muscles from pressure of body weight as well as to increase the self-muscular massage effect. Hence, foam rollers are known to improve the range of motion (Couture et al., 2015).

A study reported positive effects of muscle relaxation due to fascia relaxation and muscle tone reduction from using foam rollers before exercise in juvenile male squash athletes (Murray et al., 2016). A paper reported that foam rollers induced muscle relaxation and increased range of motion in university athletes (Bushell et al., 2015). Also, Song et al. (2014) reported that the 16-week functional movement screen training program included foam roll- 
er showed significant improvement flexibility of trunk extension and extremities. Okamoto et al. (2014) indicated that self-myofascial release using a foam roller would reduce arterial stiffness. A study reported that the group that used foam rollers had about 6\% better vertical jump and improved muscle activation of the knee extensor muscle than the control group (MacDonald et al., 2013).

Due to the stiffness of hamstring, landing after vertical jumping would cause hamstring injuries. In particular, the greater the knee extension angle in vertical jumping, the lower the risk of hamstring injury. Therefore, the increase in the knee extension angle causes a greater torque at hamsting, thereby reducing the load on the knee and preventing the injury (Brockett et al., 2004).

Recently, foam rollers combined with vibration have been widely used. Vibration is safe method to increase bone density. The several studies reported the increase of the range of motion by inducing muscle contraction and relaxation rapidly and decreasing abnormal muscle contraction and relaxation through stimulation of the Golgi-tendon organ (Colloca and Keller, 2004; Rittweger et al., 2000).

Although the whole body vibration motion, sling, and local vibration stretching method are widely introduced, research on foam rollers combined with vibration is severely limited. Therefore, this study aims to investigate the immediate effect of using vibration rollers on hamstring flexibility and jumping performance.

\section{MATERIALS AND METHODS}

\section{Subjects}

Twenty college students participated in this study. They were recruited from Nambu University in Gwangju, Korea and were randomly allocated to one of two groups: the foam roller with vibration group (FRV; $\mathrm{n}=10$ ) or the foam roller with no vibration (FRNV; $\mathrm{n}=10$ ). Data on the general characteristics of the subjects are presented in Table 1. All subjects read and signed informed consent forms in accordance with the ethical standards of the Declaration of Helsinki. The sample size was analyzed using the G-power program. The effect size was 0.6 , the significance level was 0.05 , the power was $70 \%$, and the number of group was 2 . The minimum number of samples was 20 .

The selection criterion was as follows: no neurological or orthopedic diseases that may affect intervention; not taking muscle relaxant drugs. The exclusion criteria were as follows: any prior participation in studies similar to this study within the past 1 year; and greater than $33 \mathrm{~cm}$ in the sit to reach test (Roylance et al., 2013), those who are pregnant, kidney stones, thrombosis, infec-
Table 1. General characteristics of subjects

\begin{tabular}{lcccc}
\hline Characteristic & FRV & FRNV & $t$ & $P$-value \\
\hline Gender & & & & \\
Male & 7 & 7 & & \\
Female & 3 & 3 & & \\
Age $(\mathrm{yr})$ & $20.21 \pm 1.01$ & $21.72 \pm 2.16$ & -1.681 & 0.119 \\
Height $(\mathrm{cm})$ & $170.34 \pm 10.6$ & $170.91 \pm 8.12$ & -0.143 & 0.888 \\
Weight $(\mathrm{kg})$ & $63.67 \pm 11.10$ & $68.93 \pm 17.16$ & -0.808 & 0.430 \\
Sit and reach $(\mathrm{cm})$ & $6.77 \pm 7.40$ & $5.89 \pm 7.45$ & 0.285 & 0.779
\end{tabular}

Values are presented as mean \pm standard deviation.

FRV, foam roller with vibration; FRNV, foam roller with no vibration.

tious diseases, etc. All subjects were adequately explained about the purpose and procedure of the study.

The two groups were each supervised for their exercises that consisted of application of a foam roller to the hamstring for $1 \mathrm{~min}$ 5 times for a total of $10 \mathrm{~min}$. The exercise position set the sitting on the floor. Foam roller was placed under the hamstring for the exercise. FRV (Hyperice, VYPER 2.0, Irvine, CA, USA) and FRNV were applied on the hamstring. The duration of that was $32 \mathrm{~Hz}$.

\section{Hamstring flexibility measurement}

The active straight leg raising (ASLR) test in supine position and active knee extension (AKE) test in sitting position were performed to measure hamstring flexibility. The ASLR test was measured the angle of maximum flexion of the hip joint in supine position when the leg raised straightly. The AKE test was measured the angle of knee extension in sitting position. AKE measured the angle of knee joint until the knee joint was pulled to the hamstring when the leg was stretched on the seat. The range of joint motion of ASLR and AKE was measured using a wireless electrogoniometer (BPM pathway, Andover, Hampshire, UK). According to electrogoniometer, ASLR test was attached to the lateral epicondyle of femur, and AKE test was attached to the superior part of the lateral malleolus. The both tests were taken 3 times and the average was used.

\section{Vertical jump test}

The vertical jump method was used to measure jump performance. Subjects were asked to stand on their feet and put markers on their hands, bend the knee and hip joints, and then jumped to the maximum. It was measured length from floor to the marked point using tapeline. The highest value was taken as the data after three consecutive vertical jumps with the verbal instruction "bend your knees and go as high as possible and reach your hands against the wall'. In order to exclude the elasticity of the shoe, the bare feet 
Table 2. Comparison of outcome between FRV and FRNV

\begin{tabular}{|c|c|c|c|c|c|c|c|c|c|c|}
\hline \multirow{2}{*}{ Variable } & \multicolumn{2}{|c|}{ FRV } & \multicolumn{2}{|c|}{ Within group } & \multicolumn{2}{|c|}{ FRNV } & \multicolumn{2}{|c|}{ Within group } & \multicolumn{2}{|c|}{ Between group } \\
\hline & Pre & Post & $t$ & $P$-value & Pre & Post & $t$ & $P$-value & $F$ & $P$-value \\
\hline \multicolumn{11}{|l|}{$\operatorname{ASLR}\left({ }^{\circ}\right)$} \\
\hline Left & $74.60 \pm 9.19$ & $81.50 \pm 8.23^{a, b)}$ & -5.260 & 0.001 & $57.50 \pm 12.61$ & $59.40 \pm 11.16$ & -0.772 & 0.460 & 8.468 & 0.010 \\
\hline Right & $71.90 \pm 10.33$ & $81.90 \pm 6.26^{\mathrm{a}, \mathrm{b})}$ & -5.883 & 0.000 & $56.40 \pm 14.38$ & $63.70 \pm 10.19$ & -2.177 & 0.057 & 11.249 & 0.004 \\
\hline \multicolumn{11}{|l|}{$\operatorname{AKE}\left({ }^{\circ}\right)$} \\
\hline Left & $74.90 \pm 9.45$ & $79.90 \pm 7.41^{a, b)}$ & -3.682 & 0.005 & $58.20 \pm 8.87$ & $61.20 \pm 8.73$ & -1.526 & 0.161 & 4.973 & 0.040 \\
\hline Right & $75.45 \pm 9.31$ & $80.60 \pm 7.19^{a)}$ & -3.563 & 0.006 & $58.80 \pm 10.82$ & $65.10 \pm 9.95$ & -1.945 & 0.084 & 2.351 & 0.144 \\
\hline $\mathrm{VJ}(\mathrm{cm})$ & $257.60 \pm 23.18$ & $259.00 \pm 24.06$ & -2.090 & 0.066 & $256.40 \pm 18.61$ & $257.60 \pm 18.73$ & -1.177 & 0.269 & 0.021 & 0.887 \\
\hline
\end{tabular}

Values are presented as the mean \pm standard deviation.

FRV, foam roller with vibration; FRNV, foam roller with no vibration; ASLR, active straight leg raising; AKE, active knee extension; VJ, vertical jump.

a) Significant difference between pre and post. ${ }^{b}$ Significant difference between FRV and FRNV groups.

were measured on a flat floor.

\section{Statistical analysis}

All analyses were performing using IBM SPSS Statistics ver. 22.0 (IBM Co., Armonk, NY, USA). All measurements are reported as the mean \pm standard deviation. Independent $t$-test was used to compare the general characteristics of subjects, such as age, height, and weight between the FRV and FRNV groups. A comparison with two groups was analyzed using analysis of covariance. The pretest values of each group were used as covariates for adjustment. The statistical significance level was set to $\alpha=0.05$.

\section{RESULTS}

Among the general characteristics of participants, age, height, weight, and sit-and-reach result did not show any significant difference between the two groups (Table 1).

ASLR after the intervention in the FRV group was significantly increased in both legs $(P<0.05)$ (Table 2). AKE after the intervention was significantly increased in both legs $(P<0.05)$ (Table 2$)$. The vertical jump score after intervention was not significantly different compared with before the intervention $(P>0.05)$.

The results of ASLR in the FRV group were significantly increased in both legs compare with the FRNV group. The results of AKE in the FRV group were significantly increased in only the left leg compare with the FRNV group. The height of the vertical jump was not significantly different between the two groups.

\section{DISCUSSION}

Many studies have shown that exercises that stretch the hamstring have a positive effect on the prevention of injury and reha- bilitation. The hamstring tends to be adaptive shortening that lack flexibility for a long period of sitting accompanied by lower vertebral body shortening. This may cause back pain and reduce performance.

Especially, due to decreasing flexibility of hamstring, it may cause the hamstring injury when landing after the vertical jump. Larger the angle of knee extension when vertical jumping, the lower the risk of hamstring injury. Therefore, an increase in the angle of knee extension may create a greater torque at the hamstring, thereby reducing the load on the knee, thus preventing injury. A reporter examined the relationship between flexibility of the hamstring and muscle performance (Kalichman and Ben David, 2017). They found that when the flexibility of hamstring is improved, the maximum eccentric torque of $60^{\circ}, 120^{\circ}$ and maximum concentric torque of $120^{\circ}$ is increased.

Foam rollers before and after exercising have been used to improve flexibility of the hamstring and performance. The purpose of this study was to compare the immediate effect of application of foam rollers with or without vibration on hamstring flexibility and jumping performance.

The hip flexion angle of ASLR in the FRV group was significantly improved after the intervention. The angle of knee joint extension of the AKE test in the FRV group was significantly improved after the intervention. The FRV group showed an increase in both ASLR and AKE after the intervention compared with the FRNV group. This indicates that FRV may help improve flexibility of the hamstring.

FRV as a self-fascia relaxation tool is one of the easiest ways to relieve pain and maintain flexibility in the muscles and fascia. Fascia relaxation regulates arterial function and vascular endothelial function, as well as the autonomic nervous system (Kalichman and Ben David, 2017). Vibration would accelerate muscle con- 
traction and relaxation, thereby reducing abnormal muscle contraction, stimulating the organs of the tendon to induce relaxation, and improve the range of motion (Colloca and Keller, 2004). Tonic vibration reflex causes activation of the sensory nerve fibers, such as Ia fibers and II fibers in the muscle spindles and reflexive contraction occurs by alpha motor neurons (Dallas et al., 2015). Tonic vibration reflex quickly shortens the length of the muscles, resulting in increased susceptibility to involuntary muscle contraction and relaxation. Vibration stimulation was considered to have a positive effect on the extension range of motion of the knee with relaxation of the hamstring.

In this study, a vibration stimulus applied to the foam roller was $32 \mathrm{~Hz}$. A vibration of below $20-\mathrm{Hz}$ causes excessive relaxation; vibration above $50-\mathrm{Hz}$ causes muscle soreness; and vibration of 20 $50 \mathrm{~Hz}$ would be therapeutic (Rittweger, 2010). The most effective vibration stimulation for muscle activation is 30 to $50 \mathrm{~Hz}$ (Luo et al., 2005). A study reported improvement of the range of motion and pain in the hip joint when vibration foam roller was applied with a vibration of $30-50 \mathrm{~Hz}$ (Luo et al., 2005). The FRV improved the range of motion of the ankle joint and decreased pain for university students (Cheatham et al., 2017; Han et al., 2017). The activation of the muscles seems to increase the flexibility by enhancing the muscular strength and the involuntary relaxation sensitivity by tonic vibration reflex.

In the vertical jump performance test, the jump performance was increased in both groups, but there was no significant difference. Because vertical jumping requires instantaneous power of several muscles, it can be influenced by other muscle conditions besides the hamstring. The most affected muscles in the vertical jumping were the extensor muscles of each joint (Nuzzo et al., 2008). Hence, it is possible for not only the knee extensor muscles but also the ankle joint and hip joint muscles to be affected. A study suggested that foam rollers did not change the vertical jump more significantly than dynamic stretching (Hough et al., 2009). The application of only one part of the hamstring did not show a significant change in the jump height without considering the muscle size (Axel et al., 2018), muscle strength (Alemdaroğlu, 2012), and arm usage (Lees et al., 2004).

The limitation of this study was that it did not take into account the length of the calf muscle, which could affect the flexibility of the hamstring. The ankle was placed in a comfortable state for the subject, and AKE and ASLR tests were performed. The difference in the length of the calf muscle of each subject may have caused various measurements. Future studies will be necessary to compare the effects of long-term application and the ap- propriate application duration.

\section{CONFLICT OF INTEREST}

No potential conflict of interest relevant to this article was reported.

\section{REFERENCES}

Alemdaroğlu U. The relationship between muscle strength, anaerobic performance, agility, sprint ability and vertical jump performance in professional basketball players. J Hum Kinet 2012;31:149-158.

Axel TA, Crussemeyer JA, Dean K, Young DE. Field test performance of junior competitive surf athletes following a core strength training program. Int J Exerc Sci 2018;11:696-707.

Bishop D. Warm up I: potential mechanisms and the effects of passive warm up on exercise performance. Sports Med 2003;33:439-454.

Brockett CL, Morgan DL, Proske U. Predicting hamstring strain injury in elite athletes. Med Sci Sports Exerc 2004;36:379-387.

Bushell JE, Dawson SM, Webster MM. Clinical relevance of foam rolling on hip extension angle in a functional lunge position. J Strength Cond Res 2015;29:2397-2403.

Cheatham SW, Stull KR, Kolber MJ. Comparison of a vibrating foam roller and a non-vibrating foam roller intervention on knee range of motion and pressure pain threshold: A randomized controlled trial. J Sport Rehabil 2017;28:39-45.

Colloca CJ, Keller TS. Active trunk extensor contributions to dynamic posteroanterior lumbar spinal stiffness. J Manipulative Physiol Ther 2004;27:229-237.

Couture G, Karlik D, Glass SC, Hatzel BM. The effect of foam rolling duration on hamstring range of motion. Open Orthop J 2015;9:450-455.

Dallas G, Paradisis G, Kirialanis P, Mellos V, Argitaki P, Smirniotou A. The acute effects of different training loads of whole body vibration on flexibility and explosive strength of lower limbs in divers. Biol Sport 2015;32:235-241.

Han SW, Lee YS, Lee DJ. The influence of the vibration form roller exercise on the pains in the muscles around the hip joint and the joint performance. J Phys Ther Sci 2017;29:1844-1847.

Hough PA, Ross EZ, Howatson G. Effects of dynamic and static stretching on vertical jump performance and electromyographic activity. J Strength Cond Res 2009;23:507-512.

Kalichman L, Ben David C. Effect of self-myofascial release on myofascial pain, muscle flexibility, and strength: a narrative review. J Bodyw Mov Ther 2017;21:446-451.

Kuruma H, Takei H, Nitta O, Furukawa Y, Shida N, Kamio H, Yanagisa- 
wa K. Effects of myofascial release and stretching technique on range of motion and reaction time. J Phys Ther Sci 2013;25:169-171.

Lees A, Vanrenterghem J, De Clercq D. Understanding how an arm swing enhances performance in the vertical jump. J Biomech 2004;37:19291940.

Luo J, McNamara B, Moran K. The use of vibration training to enhance muscle strength and power. Sports Med 2005;35:23-41.

MacDonald GZ, Penney MD, Mullaley ME, Cuconato AL, Drake CD, Behm DG, Button DC. An acute bout of self-myofascial release increases range of motion without a subsequent decrease in muscle activation or force. J Strength Cond Res 2013;27:812-821.

Murray AM, Jones TW, Horobeanu C, Turner AP, Sproule J. Sixty seconds of foam rolling does not affect functional flexibility or change muscle temperature in adolescent athletes. Int J Sports Phys Ther 2016;11:765776.

Nuzzo JL, McBride JM, Cormie P, McCaulley GO. Relationship between countermovement jump performance and multijoint isometric and dynamic tests of strength. J Strength Cond Res 2008;22:699-707.

Okamoto T, Masuhara M, Ikuta K. Acute effects of self-myofascial release using a foam roller on arterial function. J Strength Cond Res 2014;28: 69-73.

Peacock CA, Krein DD, Antonio J, Sanders GJ, Silver TA, Colas M. Com- paring acute bouts of sagittal plane progression foam rolling vs. frontal plane progression foam rolling. J Strength Cond Res 2015;29:23102315.

Rittweger J. Vibration as an exercise modality: how it may work, and what its potential might be. Eur J Appl Physiol 2010;108:877-904.

Rittweger J, Beller G, Felsenberg D. Acute physiological effects of exhaustive whole-body vibration exercise in man. Clin Physiol 2000;20:134142.

Roylance DS, George JD, Hammer AM, Rencher N, Fellingham GW, Hager $\mathrm{RL}$, Myrer WJ. Evaluating acute changes in joint range-of-motion using self-myofascial release, postural alignment exercises, and static stretches. Int J Exerc Sci 2013;6:310-319.

Schroeder AN, Best TM. Is self myofascial release an effective preexercise and recovery strategy? A literature review. Curr Sports Med Rep 2015; 14:200-208

Škarabot J, Beardsley C, Štirn I. Comparing the effects of self-myofascial release with static stretching on ankle range-of-motion in adolescent athletes. Int J Sports Phys Ther 2015;10:203-212.

Song HS, Woo SS, So WY, Kim KJ, Lee J, Kim JY. Effects of 16-week functional movement screen training program on strength and flexibility of elite high school baseball players. J Exerc Rehabil 2014;10:124-130. 